\begin{tabular}{|c|l|}
\hline Title & Electromyographic Characterization of Walking Behavior Initiated Spontaneously in Crayfish \\
\hline Author(s) & Chikamoto, Kyoko; Kagaya, Katsushi; Takahata, Masakazu \\
\hline Citation & $\begin{array}{l}\text { Zoological Science, 25/8), 783-792 } \\
\text { https:/doi.org/L0.2108/2s.25.783 }\end{array}$ \\
\hline Issue Date & 2008-08 \\
\hline Doc URL & http://hdl.handle.net/2115/39624 \\
\hline Rights & (c) Zoological Society of Japan /本文献の公開は著者の意思に基づくものである \\
\hline Type & article \\
\hline File Information & zsj25p783.pdf \\
\hline
\end{tabular}

Instructions for use 


\title{
Electromyographic Characterization of Walking Behavior Initiated Spontaneously in Crayfish
}

\author{
Kyoko Chikamoto', Katsushi Kagaya ${ }^{1}$ and Masakazu Takahata ${ }^{1,2 *}$ \\ ${ }^{1}$ Division of Biological Sciences, Graduate School of Science, Hokkaido University, \\ Sapporo 060-0810, Japan \\ ${ }^{2}$ Department of Life Sciences, Faculty of Science, Hokkaido \\ University, Sapporo 060-0810, Japan
}

\begin{abstract}
Crayfish initiate walking behavior not only reflexively in response to external stimuli but also spontaneously in the absence of any specific stimulus. In order to analyze the initiation mechanism underlying these different types of walking, we made simultaneous electromyographic (EMG) recordings from thoracic legs when animals initiated walking, either reflexively or spontaneously, and video recorded their movements synchronously with the EMG recording. Two different stimuli, mechanical and chemical, were used to reflexively induce walking. A non-rhythmic, sustained activation of leg muscles was found to precede the behavioral initiation of either type of walking. The duration of this non-rhythmic muscle activation was significantly longer in the spontaneously initiated walking than in the mechanical stimulus-evoked walking, although no difference was observed between the spontaneous and chemical stimulus-evoked walking. EMG recordings from all eight legs revealed that their non-rhythmic muscle activation occurred almost simultaneously prior to initiation of rhythmical stepping movements. When an animal was suspended without a leg substratum, the timing of muscle activation was more variable among the legs than in the free condition on the substratum. When the circumesophageal commissures were both severed to eliminate signals descending from the brain to the thoracic ganglia, the bilaterally coordinated rhythmic burst activity was not observed in the walking legs. These findings suggest that the spontaneous initiation of walking behavior requires sensory feedback signals from leg proprioceptors, subserved by a different descending activation mechanism from that for stimulus-driven initiation of walking.
\end{abstract}

Key words: crayfish, electromyogram, spontaneous behavior, reflexive behavior, locomotion

\section{INTRODUCTION}

Animals initiate their behavior not only reflexively in response to external stimuli but also spontaneously in the absence of any specific stimulus (Maye et al., 2007). The neurophysiological mechanisms underlying sensory-evoked behavior have been intensively analyzed, especially in invertebrates, at the level of identified nerve cells (e.g., Edwards et al., 1999; Blitz and Nusbaum, 2007). In the most common scheme (Kristan and Gillette, 2007), external stimuli are detected by sensory receptors and transmitted to sensory processors where relevant information is filtered and integrated. The output of sensory processors is then fed to command neurons so that a specific pattern generator controlling the spatio-temporal firing of motor neurons is selected and activated for a specific behavioral act. Neuronal organization of these central networks, including the sensory processors, command neurons, and pattern generators, has been intensively studied in many invertebrates (Marder et al., 2005; Katz and Hooper, 2007).

\footnotetext{
* Corresponding author. Phone: +81-11-706-2749; Fax : +81-11-706-4923; E-mail: takahata@sci.hokudai.ac.jp
}

The neurophysiological mechanism of spontaneous initiation of behavior, in contrast, remains largely unknown in invertebrate animals despite the extensive accumulation of experimental data on central neurons and their networks. Davis et al. (1974) posited that when an animal decides to do a certain behavior, the command neurons for that behavior are activated while those for all other behaviors are inhibited. A number of studies have been done to demonstrate the existence of command neurons for a variety of behavioral acts in annelids, mollusks, and arthropods (Kristan et al. 2005; Clarac and Pearlstein, 2007) by testing whether these neurons can elicit a specific behavior upon stimulation and are active during the behavior. A question here is whether or not a specific set of command neurons is activated when an animal initiates a specific behavior spontaneously, and if so, how they are activated in the absence of sensory information. Is there any possibility that different sets of command neurons are activated depending on whether the animal initiates a specific behavior reflexively or spontaneously? There is little experimental evidence for dealing with these questions.

The crayfish Procambarus clarkii was the first animal shown to have command neurons in its central nervous system: a single action potential in any of two pairs of giant fibers, called "command" neurons by Wiersma (1947), could cause the crayfish to execute a tailflip escape response. In 
the circumesophageal commissure, Bowerman and Larimer (1974) isolated small bundles of axons and stimulated them electrically to see whether any specific behavior would occur upon stimulation. These experiments finally led to the functional identification in the commissure of more than 121 command neurons for corresponding behaviors, including forward and backward walking. Crayfish can initiate walking either reflexively or spontaneously (Yamane and Takahata, 2002); however, no information is available on how the two types of walking are represented by activities of these command neurons in the crayfish central nervous system.

In the present study, we made electromyographic (EMG) recordings from the mero-carpopodite and coxobasopodite muscles of the thoracic legs and simultaneously video recorded leg movements to quantitatively characterize walking behavior initiated either reflexively or spontaneously. We also analyzed the EMG activity patterns in spontaneously initiated walking under various experimental conditions to examine what kind of information was required for the behavior. The results suggested that the spontaneous initiation of walking behavior requires sensory feedback signals from leg mechanosensory organs, subserved by a different descending activation mechanism from that for stimulus-driven initiation of walking.

\section{MATERIALS AND METHODS}

\section{Animals and preparations}

Adult crayfish (Procambarus clarkii Girard) of both sexes (8$9.5 \mathrm{~cm}$ in body length from rostrum to telson) were used in this study. They were obtained commercially and maintained in laboratory tanks, fed weekly on a diet of chopped potato and liver. Prior to the experiments to compare spontaneous with the stimulusevoked walking, they were fed chopped liver once every three days for two weeks. They were then given nothing to eat for three days preceding to the experimental trials to keep their motivational state as constant as possible.

In all experiments except those for examining the effects of descending brain signals on walking behavior, no surgical operation was made to the animal body, except in electromyographic (EMG) recording from walking legs. Extracellular recording from one leg muscle was made through a pair of $125-\mu \mathrm{m}$ silver wires coated with Teflon except at the cut tip. A pair of fine holes was drilled through the cuticle, and the electrode tips were inserted against a relatively immobile region of the muscle. The holes were sealed and the wires were fixed to the cuticle with glue. All appendages, including the chelipeds, remained intact.

A typical walking leg is depicted in Fig. 1A. Eight muscles produce the movements of the coxopodite and basipodite (Ayers and Davis, 1977). In this study, electromyograms were recorded from four leg muscles: the mero-carpopodite flexor (MCF), merocarpopodite extensor (MCE), coxo-basipodite depressor (CBD), and coxo-basipodite elevator (CBE). Since the musculature arrangement of the meropodite is complicated (Bush et al., 1978), we always had to be careful about recording contamination from muscles other than the targeted one. In some experiments, activities of other muscles were concurrently recorded with the target muscle activities to confirm the origin of the intended recording. All eight walking legs, i.e., the second to fifth pairs legs, were used for EMG recordings. The chelipeds mainly remained immobile during locomotion and therefore were not included in this study.

\section{Experimental apparatus and stimulation}

In the experiment to compare muscle activity during spontaneous and stimulus-evoked walking, a crayfish was placed indi- vidually into an aquarium $(35 \mathrm{~cm}$ long $\times 16 \mathrm{~cm}$ wide $\times 22 \mathrm{~cm}$ high). The bottom was covered with a sheet of plastic net that prevented the crayfish from slipping on the tank floor during walking. The tank was filled with $7 \mathrm{~cm}$ of water. A video camera (GZ-MG70, Victor), filming at a rate of 30 frames per second, was used to record the movement of crayfish (Fig. 1B). The area covered by the camera was $20 \mathrm{~cm} \times 35 \mathrm{~cm}$. An LED was driven periodically by electric signals that were simultaneously fed to a computer through an A/D converter to synchronize video frames with EMG recordings.

In other experiments to investigate leg muscle activities during spontaneous movement under three different conditions, a round tank (43 cm in diameter, $14 \mathrm{~cm}$ high) was used in addition to the one described above. Recording was done with crayfish in the following conditions: (1) free to move, and (2) restrained with or (3) without a leg substratum. The bottom of the tank was covered with sand grains to prevent the animals from slipping during walking. In the first condition, the crayfish could move freely in the round tank filled with $7 \mathrm{~cm}$ of water. In the second and third conditions, a steel nut was glued on the dorsal surface of the carapace so that the animal could be held under the water of the experimental aquarium or in the air.

Two different stimuli, mechanical and chemosensory, were used to exogenously induce walking. For mechanical stimulus, the tail fan was touched manually with a soft brush for a short time $(<1 \mathrm{sec})$. As the chemosensory stimulus, a piece of liver was placed manually in front of the animal. It was placed on the bottom of the tank through an acrylic glass cylinder $(38 \mathrm{~cm}$ long, $1.8 \mathrm{~cm}$ in diameter) that was dipped beforehand in the water to a depth of $2 \mathrm{~cm}$ from the bottom in order to minimize water disturbance. The liver piece was placed 5 to $10 \mathrm{~cm}$ ahead of the animal's body or alongside it at a distance of about $5 \mathrm{~cm}$. The timing of both stimuli was determined by the video frame and, when applicable, stimulus artifacts due to capacitative noises on the EMG recordings.

\section{Data storage and processing}

Signals from the extracellular electrodes for recording leg muscle activities were differentially amplified (Nihon-Kohden, MEG2100), displayed on an oscilloscope (Tektronix 5115), and digitized at $1 \mathrm{kHz}$ by using an $\mathrm{A} / \mathrm{D}$ converter (Power Lab, AD Instruments) and the associated software (Chart version 4.2, AD Instruments).

The crayfish leg consists of seven segments and seven joints, but the movements during locomotion mainly involve three joints: anterior-posterior movements involve the thoraco-coxopodite (T-C) joint, whereas leg-raising movements are performed by the coxobasipodite (C-B) joint. The mero-carpopodite (M-C) joint is responsible for lateral extension/flexion movements. In the video analysis, the distal position of the leg was represented by the dactylopodite tip and the proximal position by the T-C joint. These two positions were plotted for each leg at the rate of 30 frames per second (Fig. 1C). The change in the distance from the dactyl to the T-C joint indicates lateral extension/flexion movements; an increase in this parameter shows the forward movement (return stroke), whereas a decrease shows the backward movement (power stroke), of the whole leg. Leg movement during walking was therefore characterized by successively analyzing these two positions.

\section{Determination of the timing of muscle activity change}

In some recordings, it was practically difficult to determine exactly when the muscle activity changed when the animal initiated walking behavior. Since the muscle activity could be regarded to change from the resting or inactive state to the walking or active state when the animal initiated walking behavior, we adopted in this study the minimum AIC (Akaike information criterion) procedure to objectively determine when the state transition occurred, i.e., the time of leg muscle activation. Theoretical details of the procedure are provided in a paper by Kitagawa and Akaike (1978). Briefly, in non-stationary time-series data in which the state shows a transi- 
A
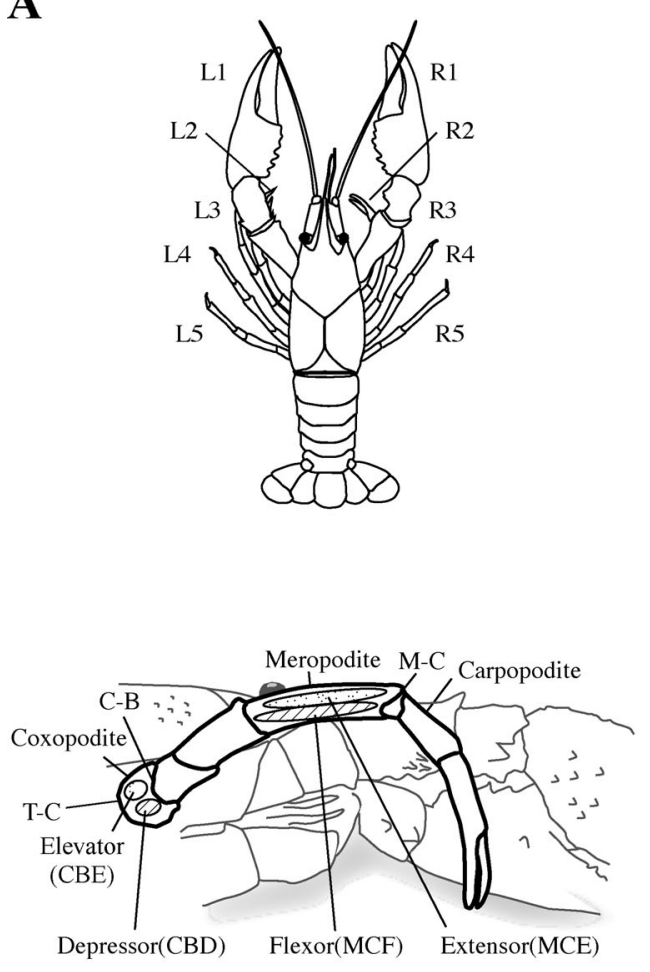

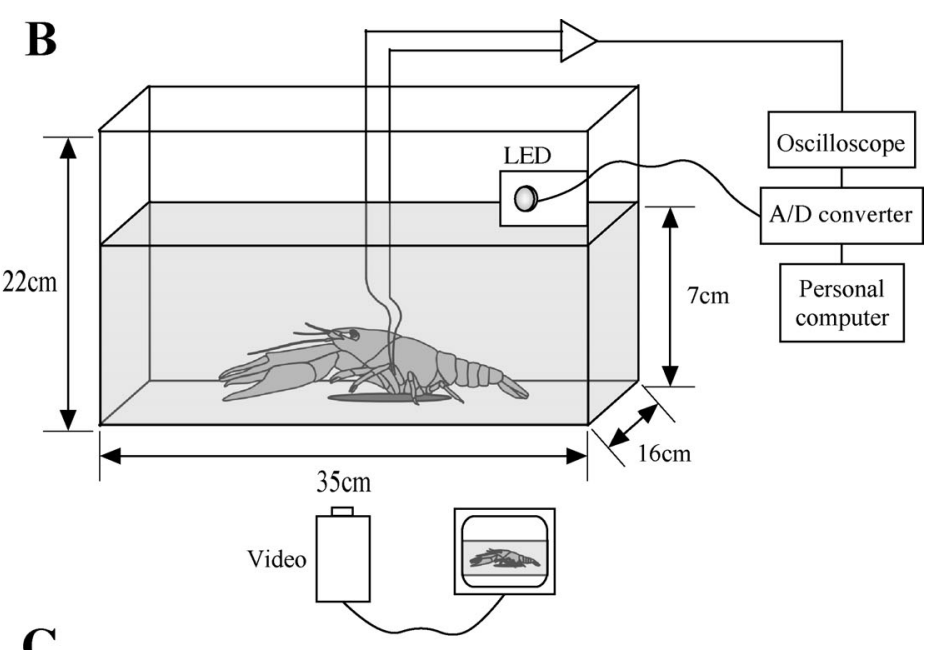

C

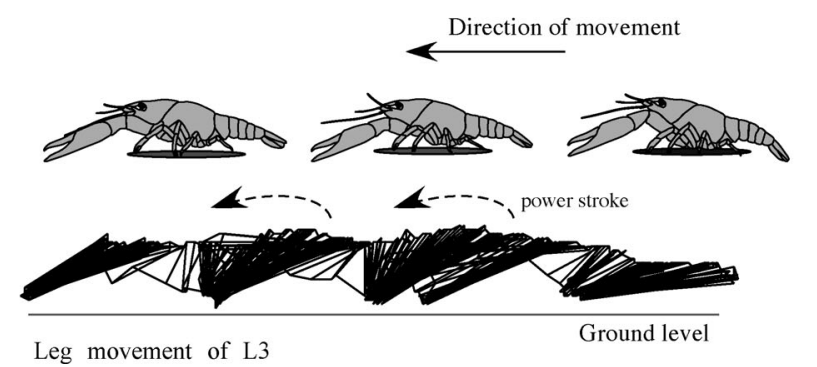

Fig. 1. Experimental apparatus and procedures. (A) The thoracic walking legs of crayfish comprise five pairs of pereopods (L1-L5 and R1R5). The first pair (L1 and R1) is transformed into large chelae and is generally not used in walking, whereas the second and third pairs having smaller pincers are actively used. Shown below is a posterior-lateral view of the fourth right leg and major muscle groups. Each leg is composed of seven segments. The first (thoraco-coxopodite), second (coxo-basipodite), and fifth (mero-carpopodite) joints are crucial for walking, allowing the leg to move in the forward/backward and upward/downward directions, and to extend/flex, respectively. C-B, coxo-basipodite; MC, mero-carpopodite; T-C, thoraco-coxopodite; CBD, coxo-basipodite depressor; CBE, coxo-basipodite elevator; MCE, mero-carpopodite extensor; MCF, mero-carpopodite flexor. (B) Experimental aquarium and recording apparatus. A crayfish was placed in an aquarium in which the bottom was covered with a sheet of plastic net, containing water to a depth of $7 \mathrm{~cm}$. An LED was driven by electrical pulse signals that were fed to an A/D converter and then to a personal computer, simultaneously with the EMG signals. A video camera was placed by alongside the crayfish. (C) Lateral view of the third left leg trajectory in successive strides. The leg movements during walking were filmed at a speed of 30 frames per second. A line was drawn in each successive frame, connecting the T-C joint and the distal end of the dactylopodite to represent the position and direction of the leg. All 550 successive frames are shown here; the movement progressed from right to left. The upper contour represents successive positions of the body joint (T-C); the bottom one, successive positions of the distal part of the leg (dactyl). The motor program for walking consists of return strokes and power strokes of walking leg movements. The fan shapes indicate the power stroke with the dactyl on the ground.

tion, any block of sufficiently short period can be regarded as stationary. Applying the locally stationary autoregressive model to each block, one can test whether any two successive blocks are in different states by calculating the AIC: the time of transition can be determined as the time that shows the minimum AIC value to separate two blocks. In this study, a period of 2 seconds, at the center of which was the putative time for the muscle activation, was chosen from the continuous EMG data. These 2-sec time-series data were analyzed by the minimum AIC procedure (Fig. 2D) to identify the time of change from the resting to the active state.

\section{RESULTS}

\section{Antagonistic muscle activities at the spontaneous initia-} tion of walking

To confirm the basic activity pattern of leg muscles in spontaneously initiated walking, EMG recordings were made from two pairs of antagonistic muscles of the third thoracic leg (Fig. 2A, B). The mero-carpopodite flexor (MCF) is antagonistic to the mero-carpopodite extensor (MCE), and the coxobasipodite depressor (CBD) to the coxo-basipodite elevator
(CBE). Each pair of antagonistic muscles showed a reciprocal activation pattern, bursting alternately during walking behavior (black-colored parts in Fig. 2A, B). A non-rhythmic, sustained increase was also observed simultaneously in both of each antagonistic pair (gray-colored parts in Fig. 2A, B) before initiation of the rhythmic bursting activities that corresponded to stepping leg movements during walking.

Similar sustained activation preceding the rhythmic bursts and stepping leg movements was also observed in the EMG recording from the coxal protractor (data not shown), one of the muscles producing the movements of the coxopodite and basipodite (Ayers and Davis, 1977). The data showed that the sustained activation preceding the rhythmic burst activities was common to many, if not all, leg muscles at the time of spontaneous initiation of walking. Similar sustained co-activation of antagonistic and agonistic muscles was also observed when the animal initiated walking in response to external stimuli, but it was quantitatively different from that observed at the time of spontaneous ini- 
A

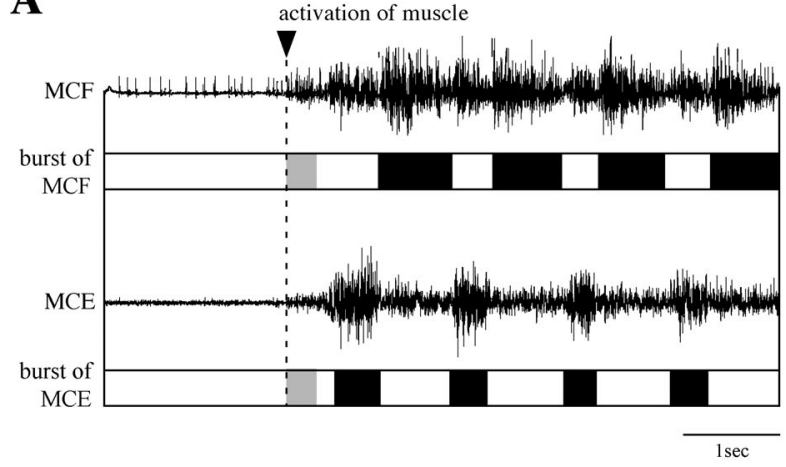

B

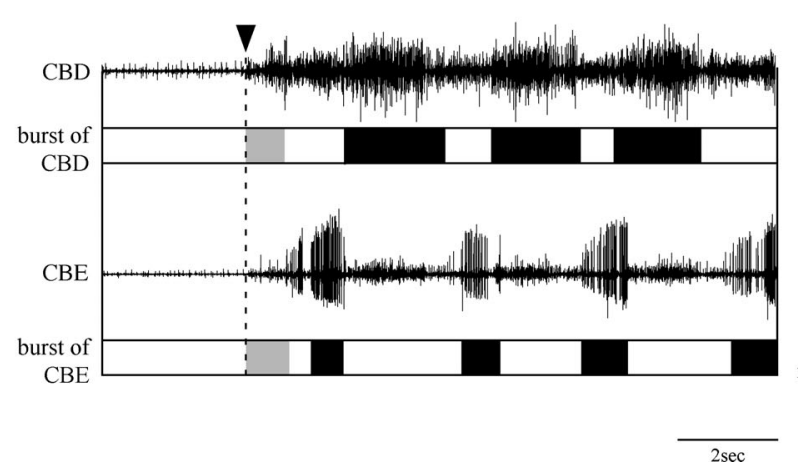

C

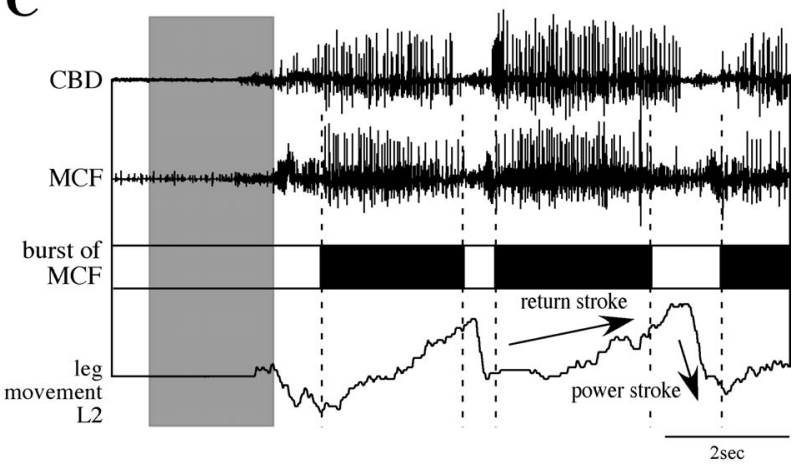

D

activation of CBD

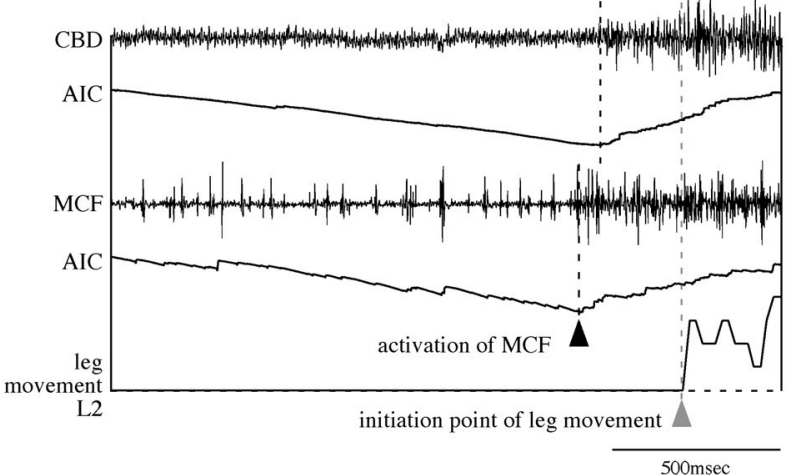

Fig. 2. Antagonistic muscle activities and leg movements. (A) Electromyograms recorded at the onset of walking from the mero-carpopodite flexor (MCF) and the mero-carpopodite extensor (MCE), which are antagonistic to each other. (B) Electromyograms recorded from the coxobasipodite depressor (CBD) and coxo-basipodite elevator (CBE). Black rectangles show alternating bursts of each pair of antagonistic muscles during walking behavior. Gray parts indicate the sustained, non-rhythmical activation of muscles preceding the initiation of rhythmic stepping activities. EMG recordings were made from the third leg in A and B. (C) Simultaneous recordings of muscle activities and leg movements at the initiation of spontaneous walking. EMG recordings were from MCF and CBD of the left third leg. The lateral extension and flexion movements of the leg were measured by the distance between the dactyl tip and the T-C joint in each video frame to quantify the return and power strokes, respectively (bottom trace). During the return stroke, MCF showed rhythmic bursts (black rectangles). (D) Temporal expansion of the record indicated by the gray rectangle in C. In order to determine the activation time of leg muscles objectively, a minimum AIC procedure was applied to the EMG record (lower black arrowhead for MCF and upper black arrowhead for CBD). A sustained, non-rhythmic increase in leg muscle activity preceded the behavioral initiation of leg movements (gray arrowhead).

tiation of walking (see below).

\section{Sustained activation of leg muscles prior to stepping movements}

To quantitatively analyze the time difference between leg muscle activation and actual leg movements, we made video and EMG recordings simultaneously at the time of initiation of spontaneous walking. Electromyograms were recorded from CBD and MCF of the second and third walking legs. In the video recording, the positions of the distal end of the dactylopodite and the proximal end of the coxa, i.e., the T-C joint, were plotted for each walking leg at the rate of 30 frames per second. The distance between these two points represented the length of the whole leg projected onto the sagittal plane of the animal body, its changes indicating lateral extension/flexion movements of the leg. The motor program for walking consists of two rhythmically alternating movements of the thoracic legs, i.e., the return (forward) stroke and the power (backward) stroke. The rhythmic bursts of MCF (black-colored parts in Fig. $2 \mathrm{C}$ ) corresponded to the return stroke during stepping movements.
Close examination of the EMG and video records revealed a characteristic pattern of activation of leg muscles preceding the stepping movements. Fig. $2 \mathrm{C}$ shows the initiation phase of walking (the area shaded by a gray rectangle) in the EMG record; this is expanded temporally in Fig. 2D, together with the behavioral data on leg movements obtained from video recordings. The results from the minimum AIC analysis are also shown to indicate the activation time of leg muscles, determined objectively. Comparison of the activation time of muscles (black arrowheads in Fig. 2D) and the initiation time of leg movements (gray arrowhead in Fig. 2D) showed that sustained, non-rhythmic muscle activation preceded the behavioral initiation of stepping movements of the thoracic legs. In the record shown in Fig. 2, the muscle activity increase preceded the stepping movements by $208.7 \mathrm{msec}$ in CBD and $306.1 \mathrm{msec}$ in MCF.

We also made video and EMG recordings from the second and third left legs at the time of initiation of spontaneous walking to quantify the latent period from muscle activation to stepping movements (Fig. 3A, B). Data were obtained from 13 freely moving crayfish in total. The sustained, nonrhythmic muscle activation lasted for $305 \pm 49$ msec (mean \pm 

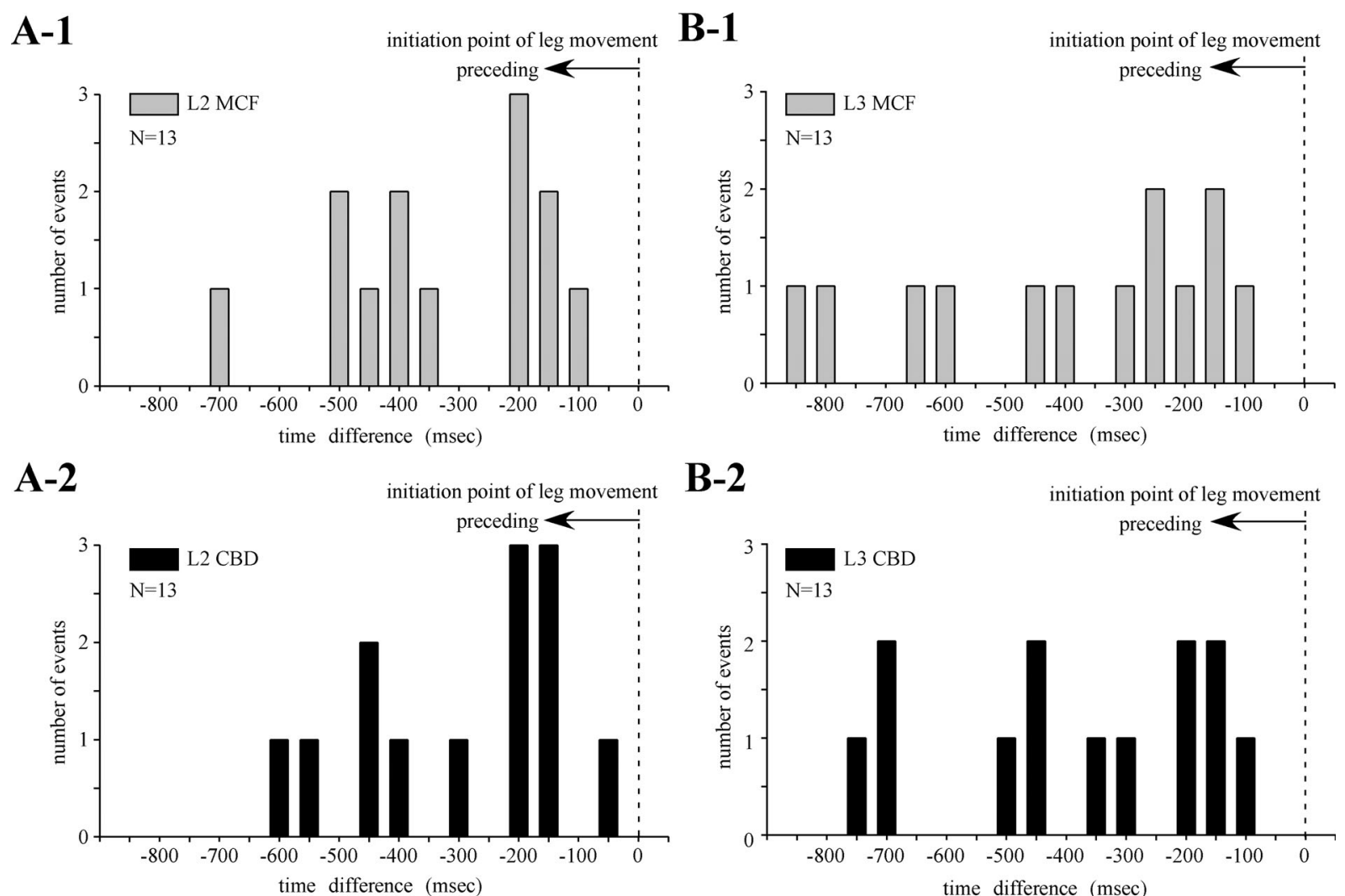

B-2

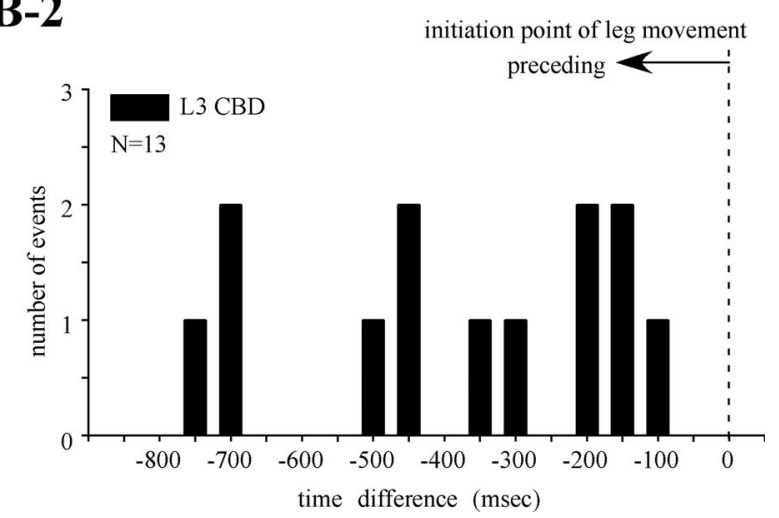

Fig. 3. Time differences between muscle activation and leg movements. (A-1) MCF and (A-2) CBD activities in the second leg. (B-1) MCF and (B-2) CBD activities in the third leg. For each panel, the non-rhythmic activation of each leg muscle prior to initiation of spontaneous walking is illustrated as a histogram with 50 -msec time bins. EMG recordings were made from six animals that spontaneously initiated walking behavior for a total of 13 times. The initiation time in each panel is indicated with a vertical dashed line.

standard error) in MCF (Fig. 3A-1) and $362 \pm 49 \mathrm{msec}$ in CBD (Fig. 3A-2) of the second left leg, and $273 \pm 70 \mathrm{msec}$ in MCF (Fig. 3B-1) and 365 $\pm 68 \mathrm{msec}$ in CBD (Fig. 3B-2) of the third leg. These results suggest that the sustained activation of leg muscles is a consistent feature of the central mechanism underlying spontaneous initiation of walking.

\section{Comparison of spontaneous and stimulus-driven walking}

We made simultaneous video and EMG recordings of walking-leg movements when animals initiated spontaneous or stimulus-evoked walking in order to compare the activation pattern of leg muscles between them. Stimulus-driven initiation of walking behavior was carried out by mechanical or chemosensory stimulation. Typical EMG activities at the onset of walking are shown in Fig. 4A, B, and C for spontaneous initiation, and mechanical and chemical stimulusdriven initiation, respectively. In all conditions, a sustained activation of MCF preceded the initiation of stepping movements for walking, although its duration varied significantly depending on how the walking was initiated.

The data for the present analyses were obtained from 33 sessions of spontaneous walking (13 animals), 23 sessions of mechanical stimulus-driven walking, and 20 sessions of chemosensory stimulus-driven walking. In this study, we could not measure the exact timing of each stimulation, since this was judged from video recording: our measurements inevitably included an error of up to $\pm 1 / 30 \mathrm{sec}$. We could, how- ever, measure exactly the time from the activation of MCF muscle (black arrowheads and dotted lines) to the initiation of walking-leg movements (gray arrowheads and dotted lines) in each trial. The mean value of time differences between MCF activation and initiation of walking was $520 \pm 325 \mathrm{msec}$ and $119 \pm 38$ msec for spontaneous and mechanical stimulusevoked walking, respectively. The difference was statistically significant (ANOVA; $p<0.0001$ ) (Fig. 4D).

When chemical stimulation evoked walking, the latent time was $439 \pm 194 \mathrm{msec}$, statistically not different from that for spontaneously initiated walking. Further experiments were carried out to examine whether or not the walking behavior induced by chemosensory stimulation involved body orientation toward the stimulus, by presenting the animal with the stimulus from different directions. Thus, the liver piece was placed $10 \mathrm{~cm}$ ahead of the rostrum or $5 \mathrm{~cm}$ to the side of the animal's body. There was no noticeable difference in the time from MCF activation to leg movement initiation or in the degree of orientation to the odor source between the two conditions, although these observations were qualitative. The chemical stimulus thus elicited walking behavior without any observable orientation of the animal body toward the stimulus source.

Effects of sensory feedback on leg-muscle activities in walking initiated spontaneously

Sensory feedback signals from leg proprioceptors have 

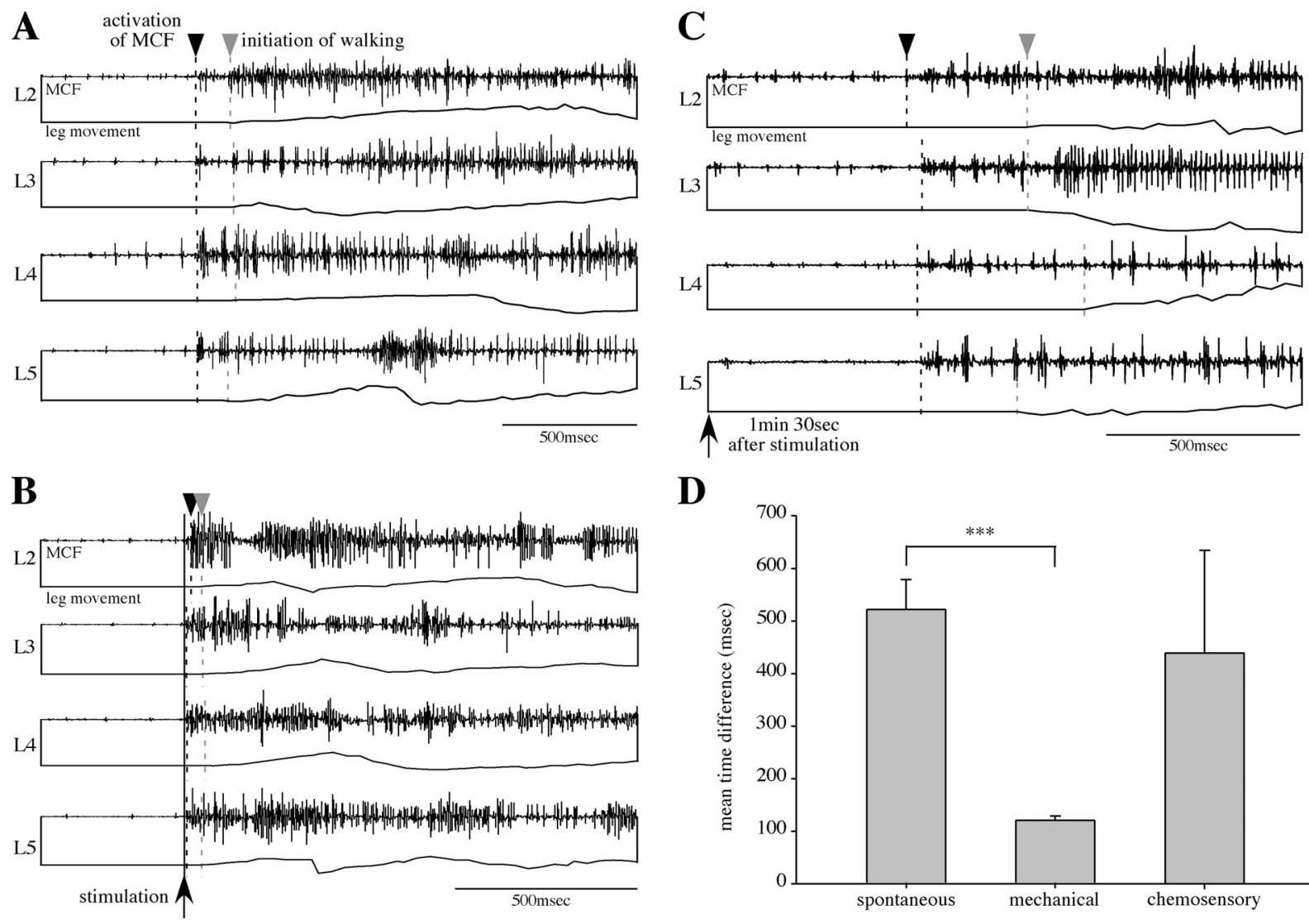

D

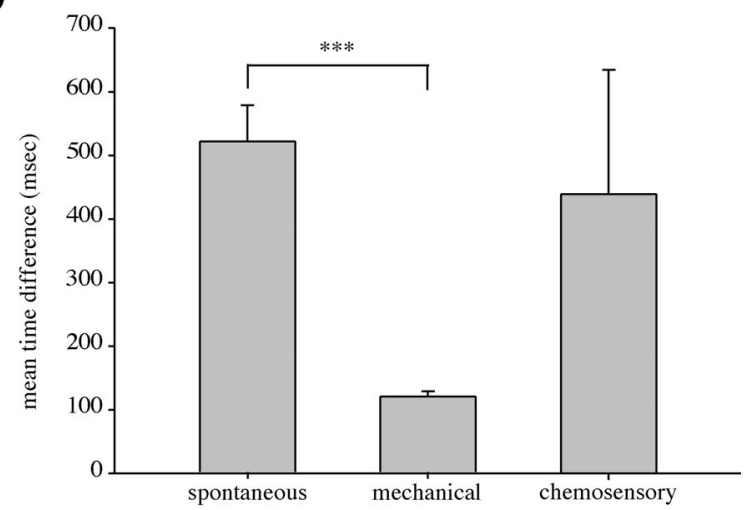

Fig. 4. Comparison between spontaneous and stimulus-evoked walking. (A) Spontaneously initiated walking. Electromyograms were recorded from MCF of the left second to fifth legs (L2-L5). The trace below each EMG record represents the leg movement obtained from video analyses, with upward deflection indicating the return stroke movement. (B) Walking initiated in response to mechanical stimulation. The vertical line (black arrow) indicates the time of stimulation. (C) Walking initiated in response to chemical stimulation. The record starts one minute and thirty seconds after the stimulation. In A-C, the black arrowhead indicates the time when the muscle showed a sustained, nonrhythmical activation preceding its rhythmical burst activities. This timing varied among legs and is indicated for each by a black dashed line. The gray arrowhead indicates the time when the leg began to show noticeable movement. This timing also varied among legs and is indicated for each by a gray dashed line. (D) Time differences between MCF activation and initiation of leg movements. The mean value for spontaneous walking was significantly greater than that for mechanical stimulus-evoked walking (ANOVA; ***p<0.0001). No significant difference was observed between spontaneously and chemically initiated walking.

been shown to be crucial for generating the coordinated stepping motor pattern in crustaceans (Le Bon-Jego et al., 2006). To investigate the effect of this feedback on leg movements when crayfish initiated walking spontaneously, we made EMG recordings from the MCF muscle of the second to fifth walking legs on both sides under three different conditions: free to move on the aquarium bottom, or restrained in water with or without a leg substratum. Electromyograms recorded under each condition are shown in Fig. 5A-C. Rhythmic and coordinated bursts in all eight legs were observed only in the free condition (Fig. 5A). Thus, the muscle was activated not only sequentially on each side but also alternately on both sides: when the MCF muscle of the second leg, for example, was activated on the left side, that on the right side was inactivated, and vice versa (Fig. 5A). In the restrained condition, neither the sequentially nor the bilaterally patterned activation of MCF not observed, irrespective of whether the leg substratum was provided (Fig. 5B) or not (Fig. 5C).

Prior to the initiation of walking, muscle activities showed a sustained increase in all eight legs almost simultaneously, within the time range of $65.89 \pm 6.40 \mathrm{msec}(\mathrm{N}=45$ from 10 animals). The muscle activation in free animals did not always lead to full walking, but the simultaneous activation of eight legs was observed irrespective of whether or not it led to walking. In the two restrained conditions, with or without the leg substratum, the increase in muscle activity occurred more irregularly, within the time range of $134.12 \pm 25.89 \mathrm{msec}$ $(\mathrm{N}=50$ from 10 animals) and $168.07 \pm 23.01 \mathrm{msec}(\mathrm{N}=42$ from 8 animals) respectively (Fig. 5D). The burst cycle duration in all eight legs, represented here as the ratio of the shortest to the longest, differed significantly between the free condition and the two restrained conditions (ANOVA, $p<0.05$; Fig. 5E). Thus, the leg movement pattern was rhythmical and bilaterally coordinated only in the free condition. The spontaneous initiation of normal walking thus could not be carried out without sensory feedback signals from leg proprioceptors, although these remained unidentified in this study.

\section{Effects of descending brain signals on the stepping motor pattern}

Surgical interruption of the descending transmission of 

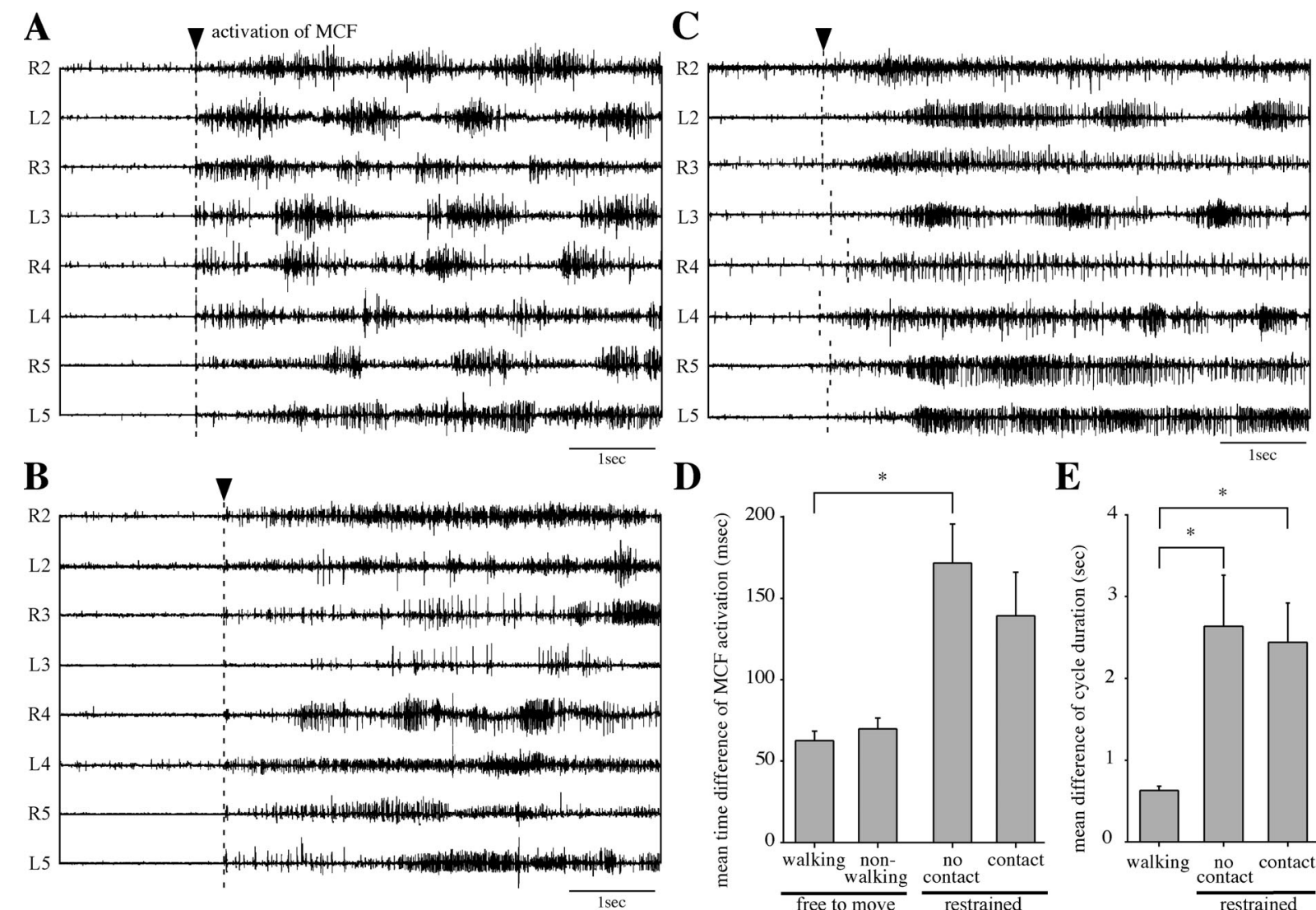

D

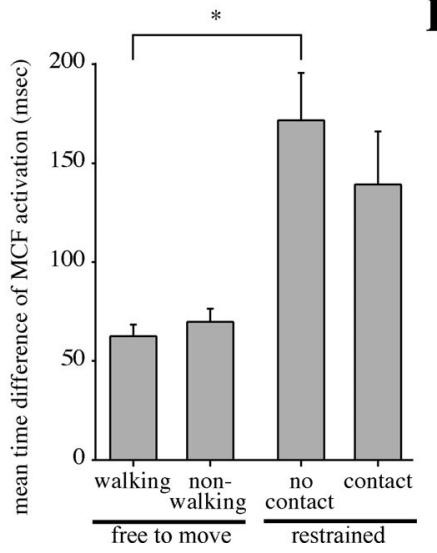

$\mathbf{E}$

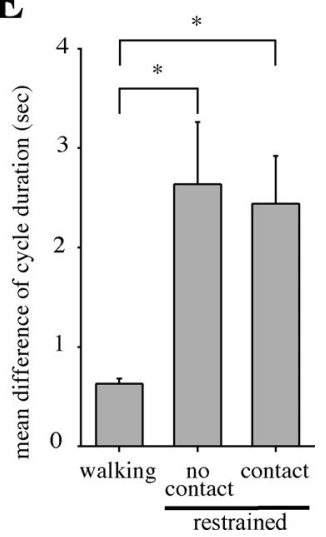

Fig. 5. Effects of sensory conditions on stepping movements. (A) EMG recordings from eight walking legs when the animal spontaneously initiated walking under the condition that it was free to move. (B) EMG recordings from the animal restrained in water, with a leg substratum. (C) EMG recordings from the animal restrained in water, without the leg substratum. The downward arrowhead and broken line represent the initiation time of MCF activation in each panel. (D) Statistical comparison of the time from MCF activation to initiation of leg movements among the different sensory conditions. In the free condition, the sustained, non-rhythmic activation began almost simultaneously in all eight legs to precede the rhythmical leg movements. The MCF activation in free animals did not always switch over to walking: it either led to rhythmical stepping behavior (walking) or aborted to non-coordinated leg movements (non-walking). The activation of MCF occurred in eight legs within a significantly longer range in the restrained condition without a leg substratum than in the free condition (ANOVA; * $p<0.05)$. (E) Statistical comparison of the cycle duration, i.e., the time from the beginning of one burst activity to that of the next, among the different sensory conditions. The comparison was based on the ratio of the shortest cycle to the longest for all legs in each data group. The burst activity, which was regularly rhythmic in the free condition, was disrupted significantly when the animal was in the restrained condition irrespective of whether or not it was provided with a leg substratum (ANOVA; * $p<0.05)(E)$.

brain signals to thoracic ganglia was found to affect the stepping motor pattern significantly. In the experiment illustrated in Fig. 6, EMG recording was made from all eight legs in animals that had their circumesophageal commissures severed bilaterally. Including control experiments, EMG recordings were made from the same animal under the following different conditions: (1) intact, i.e., with no surgical operation; (2) sham operated, i.e., after the brain and the commissures were exposed but kept intact; and (3) after the commissures were cut away. After the operation, the cephalothoracic cavity was flushed repeatedly with cooled saline to minimize damage to the nervous system caused by drying or digestive fluid. EMG recordings from the operated animals were made at least 30 minutes after the operation. For this comparison, EMGs of twenty-four walking bouts recorded from four animals were analyzed for each condition. In some cases, the activation of walking-leg muscles coincided in all legs in the third condition, although rhythmical bursts were not observed at all (Fig. 6A). The difference between the shortest and longest burst cycle duration in eight legs was not significantly different between (1) and (2), whereas it was statistically different between (1) and (3), and (2) and (3) (ANOVA, $p<0.05$; Fig. 6B). Without descending signals from the brain, the burst activities in thoracic leg muscles were thus so variable that they could not be coordinated for any purposive behavior.

Spontaneously initiated leg movements as shown in Fig. $6 \mathrm{~A}$ were seldom observed in this study after the circumesophageal commissures were severed on both sides. Their occurrence during one experimental session was much rarer than that in the intact condition during the same period. Our observations, although qualitative, did not exclude the possibility that stepping leg movements can occur spontaneously in the absence of descending signals from the brain, but strongly suggest that these movements normally require the signals under natural conditions. Further study is needed to clarify whether spontaneous leg movements in the operated condition are preceded by sustained activation of the 

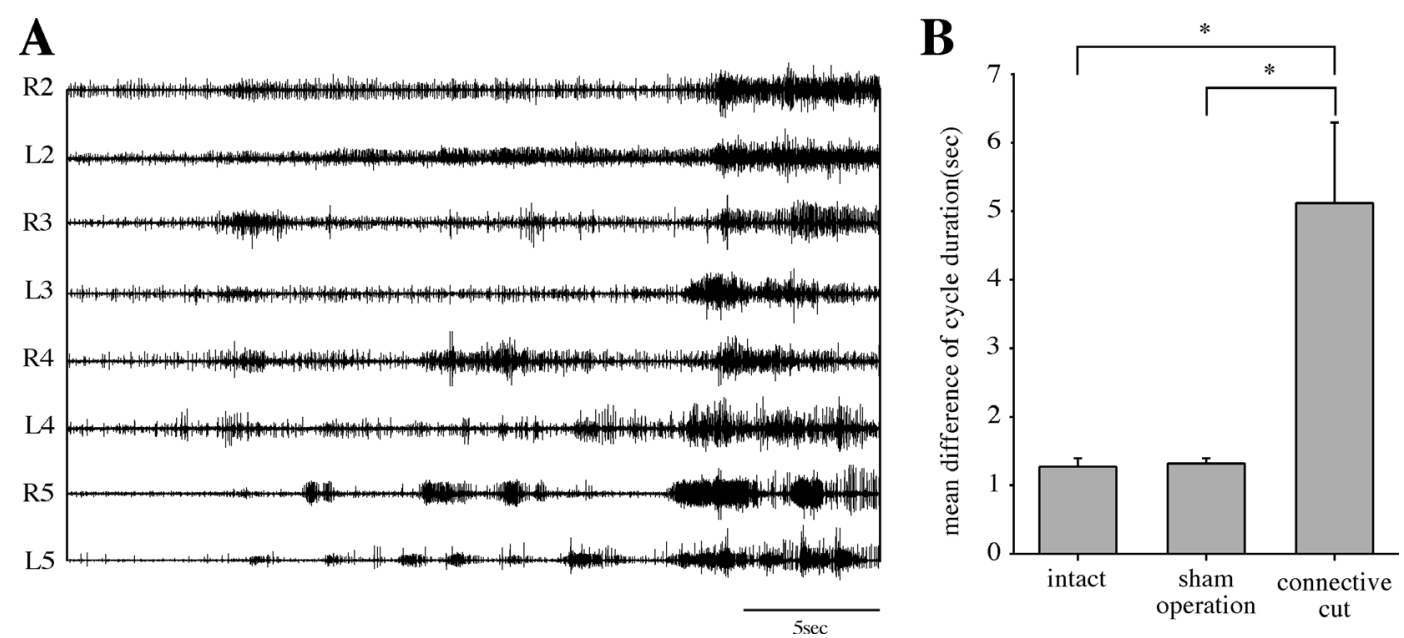

Fig. 6. Effects of descending brain signals on leg movements. (A) MCF activities in the left second to fifth legs. The EMG recordings were made when the animal was moving its legs after the operation to cut away both circumesophageal commissures, i.e., in the condition that the descending signals from the brain to the locomotor center in the thoracic ganglia were interrupted. In the later part of the record, apparent simultaneous activation of the muscle is evident in all eight legs, but any coordinated stepping activity was observed in the EMG and behavior. (B) Statistical comparison of the cycle durations, i.e., the time from the beginning of one burst activity to that of the next, among the three conditions. The comparison was based on the ratio of the shortest cycle to the longest for all legs in each data group. When the descending brain signals were interrupted, the rhythmical muscle activity could not be regulated and no stepping movement was observed in the legs (ANOVA; $* p<0.05)$.

muscles, as in the normal condition.

\section{DISCUSSION}

EMG recordings from the walking-leg muscles of freely moving crayfish revealed that sustained and non-rhythmic activation preceded the behavioral initiation of leg movements at the onset of both spontaneous and mechanical stimulus-evoked walking. The duration of this preceding muscle activity was significantly different between these two types of walking. Chemical stimulus-evoked walking was found to differ from mechanically induced walking in the duration of the preceding muscle activity. The stepping movements of legs themselves were similar among the three types of walking, but the sustained muscle activation leading to the stepping movements was thus characteristically different among them. Although EMG activity shows the extracellular junctional potentials of muscle fibers, these potentials reflect well the spike activity of motor neurons (Carew, 2000), and thus the central mechanisms subserving specific actions carried out by the recorded muscles. In the following sections, we compare the physiological processes underlying the initiation of spontaneous and stimulus-evoked walking and discuss their central nervous mechanisms.

\section{Non-rhythmic activation of leg muscles prior to walking behavior initiated spontaneously}

When crayfish initiated walking spontaneously, a sustained increase in walking-leg muscle activities was observed to precede leg movements by approximately 325 msec on average (Figs. 2D, 3A, B). The preceding muscle activity was observed almost simultaneously in the antagonistic pairs of muscles that showed alternating bursts during walking, each activated out of phase with its counterpart (Fig. 2A, B). In the locust, an increase in neural activities in the circumesophageal and neck connectives was reported to precede the initiation of spontaneous walking. This change in neural activity was regarded as the preparatory phase for the initiation of walking (Kien, 1990). The preceding activity in the leg muscles of crayfish, therefore, may also represent the preparatory activity for spontaneous walking. However, the preceding muscle activities of crayfish observed in this study are distinct from the reported activities in the locust, in that the latter lasted for several seconds and involved small leg movements that appeared to be stepping "intention" movements (Kien, 1990), whereas the former preceded the initiation of walking by a few hundred milliseconds and, more importantly, evoked no observable movements in the walking legs.

The intention movement is an incomplete behavior pattern that provides potential information that an animal is about to perform a particular activity (McFarland, 1999). It is a kind of ritualized behavior for social communication, sending signals to other animals by specific patterns in the movement of the sender's body. It is therefore imperative for any behavioral act to be clearly observable to other individuals if the act is to function as an intention movement. In this sense, the sustained activation of leg muscles preceding their stepping movements does not conform to the definition of intention movement, since this activation caused no observable behavioral activity (Fig. 2D). Furthermore, the preceding muscle activity lasted for 300-400 msec (Fig. 3A), too short for communication with other animals by behavioral means. These considerations lead us to conclude that the sustained muscle activation preceding walking behavior does not reflect any kind of intention movement, but is part of the behavior even though it is not observable in the animal's appearance. As discussed below, we interpret the preceding activation of leg muscles prior to behavioral initiation of spontaneous walking as a revelation of central nervous mechanisms. 


\section{Initiation of spontaneous and mechanical stimulus- evoked walking}

The non-rhythmic activation of leg muscles preceding walking behavior was observed regardless of whether it was initiated spontaneously or reflexively by external mechanical stimuli. The duration of this preceding muscle activity, however, varied significantly between spontaneous and mechanical stimulus-evoked walking (ANOVA, $p<0.05$; Fig. 4D). The functional role of non-rhythmic activation of leg muscles remains unknown, but the statistically significant difference in its duration suggests a qualitative difference in the neural mechanism subserving the initiation process between spontaneous and mechanical stimulus-evoked walking. Since the non-rhythmic activity preceding the rhythmic burst activities for stepping movements is smaller in amplitude than the inter-burst activity (Fig. 2C), it is not any part of the burst cycle activity of the pattern generator in the steady state, if it represents a transient form of the generated motor pattern. Our observation that some descending interneurons in the protocerebrum increase in spike activity prior to the initiation of spontaneous walking (Kagaya and Takahata, 2006) suggests that the non-rhythmic muscle activity reflects instead the central signals for activating the pattern generator.

In vertebrates, the locomotor pattern generator in the spinal cord is thought to be activated by descending signals originating from the command center located in the midbrain (Mori et al., 1991; Sirota et al., 2001; Grillner, 2006). The descending signals determine when the pattern generator is to be activated and at what level the activity is to be maintained (Orlovsky et al., 1999; Deliagina et al, 2002). In invertebrates, however, the activation mechanism for locomotor behavior remains largely unknown. Although pattern generating mechanisms have been intensively analyzed for locomotor behavior, including insect walking (Ramirez and Pearson, 1988; Burrows, 1996) and flight (Robertson and Pearson, 1985; Robertson, 1986; Pearson and Ramirez, 1997), and leech swimming (Kristan and Gillette, 2007), no information is available on the command center that would activate these mechanisms as in the vertebrate brain. In crayfish, Bowerman and Larimer (1974) reported that electrical stimulation of some units in the circumesophageal commissure could elicit forward or backward walking. It remains unknown, however, how these units are activated when the animal initiates, maintains, and terminates walking behavior. We demonstrated that descending signals from the brain are necessary for generating coordinated burst activities in the leg muscles (Fig. 6), but did not attempt in this study to analyze initiation signals for the coordinated burst activities in walking-leg muscles.

It would be safe to assume that when crayfish initiate walking behavior in response to mechanical stimulation, the locomotor pattern generator in the thoracic ganglia (Chrachri and Clarac, 1990) is activated by sensory information from the mechanical stimuli applied to the animal body. In the experiment shown in Fig. 4B, the leg muscle began to show non-rhythmic activation within $18 \mathrm{msec}$ after stimulation, reflecting the time for signal transmission from the mechanosensory neurons in the tail fan to the muscle by way of abdominal and thoracic ganglia. This activation lasted for about $41 \mathrm{msec}$ (Fig. 4B) prior to the start of rhythmic burst activities. This finding indicated that the non-rhythmic activation need not be longer, as in spontaneous initiation of walking, for the following rhythmic activities to initiate stepping behavior. A question here is, why is the non-rhythmic muscle activation preceding the stepping movements much longer for spontaneously initiated than for mechanical stimulus-evoked walking? On the basis of our EMG analysis of the process initiating stimulus-evoked walking, we hypothesize that the long duration of non-rhythmic muscle activation in spontaneous walking reflects the descending activities from the brain that are preparing for the endogenous initiation of walking behavior. Spike activities preceding volitional movements are known in the basal ganglia of the vertebrate brain (Schultz, 1999). Further study is needed to test the possibility mentioned above, but the present study revealed that, prior to the spontaneous initiation of behavior, the central nerve cells became remarkably active, although this activity was behaviorally unobservable.

\section{Initiation of chemical stimulus-evoked walking}

It usually took more than one minute for a stimulus to cause an EMG activity change. Since spontaneous walking occurred far less frequently, the stimulus was regarded to be effective in eliciting walking. Our finding that the duration of non-rhythmical muscle activation prior to behavioral initiation of chemical stimulus-evoked walking was longer than that in mechanically induced walking and statistically comparable with that in spontaneously initiated walking (Fig. 4) points to the following possibility: the relatively long duration of prebehavioral muscle activity was possibly due to a low effective concentration of chemical stimuli. A corollary to this hypothesis is the possibility that the duration of pre-behavioral muscle activation decreases as the effective concentration increases. We could not test this possibility, since it was difficult to exclude a potential mechanosensory effect when the chemical stimulus, i.e., the liver piece, was provided near the animal body. Unlike the case of mechanical stimulation, it is difficult to evaluate the effectiveness of chemical stimulation, since this is not linearly related to the source distance, concentration, or time course (Weissburg and Zimmer-Faust, 1993, 1994). Since there was no water current in the experimental aquarium, the effective stimulus strength was possibly not so high as to elicit forage walking immediately or in a short time.

It should be also noted here that the chemical stimulus eliciting walking behavior without any observable orientation toward the stimulus source is consistent with the possibility that the effective stimulus strength was not sufficient in our experiment, for it has been reported that some forms of chemically modulated orientation occur in crabs and lobsters when chemical stimuli are presented (Moore et al., 1991; Weissburg, 1997; Zimmer-Faust et al., 1995). Those experiments were made in the presence of water flow, whereas the present experiment was made in its absence; crabs and lobsters may use not only simultaneous comparison of the flow for the information contained within and between odor pulses to determine whether to remain still or turn into the plume, but also sequential comparisons of odor intensity to advance toward the source (Weissburg, 1997). It is possible that that the behavioral mechanisms underlying orientation responses to the odor source are different between animals 
(cf. Moore et al., 1991; Weissburg and Zimmer-Faust, 1993); the orientation responses of crayfish might be distinct from those of crabs and lobsters.

On the basis of our EMG recordings from animals responding to chemical stimulation, we tentatively conclude that the stimulus activated a motivational system in the crayfish brain that was common to spontaneous walking, and that depends on motivational systems for diverse behavioral acts, including foraging and feeding. Because of the low effective concentration of the chemical stimulus, this apparently acted on the motivational system instead of reflexively activating the walking motor system. Further systematic study is needed to clarify how chemical stimuli elicit walking behavior.

\section{ACKNOWLEDGMENTS}

We thank Dr. Hama for helpful comments and valuable discussions. This research was supported in part by Grants-in-Aid for Scientific Research from the Ministry of Education, Culture, Sports, Science and Technology, Japan (Nos. 17370024 and 18657025).

\section{REFERENCES}

Ayers JL, Davis WJ (1977) Neuronal control of locomotion in the lobster, Homarus americanus 1. Motor programs for forward and backward walking. J Comp Physiol 115: 1-27

Blitz DM, Nusbaum MP (2007) Mechanosensory regulation of invertebrate motor systems. In "Invertebrate Neurobiology" Ed by G North, RJ Greenspan, Cold Spring Harbor Laboratory Press, Cold Spring Harbor, pp 185-208

Bowerman RF, Larimer JL (1974) Command fibers in the circumoesophageal connectives of crayfish: II. Phasic fibres. J Exp Biol 60: $119-134$

Burrows M (1996) The Neurobiology of an Insect Brain. Oxford University Press, Oxford

Bush BMH, Vedal JP, Clarac F (1978) Inter segmental reflex actions from a joint sensory organ (CB) to a muscle receptor (MCO) in decapod crustacean limbs. J Exp Biol 73: 47-63

Carew TJ (2000) Behavioral Neurobiology: The Cellular Organization of Natural Behavior. Sinauer, Sunderland

Chrachri A, Clarac F (1990) Fictive locomotion in the fourth thoracic ganglion of the crayfish, Procambarus clarkii. J Neurosci 10: 707-719

Clarac F, Pearlstein E (2007) Invertebrate preparations and their contribution to neurobiology in the second half of the 20th century. Brain Res Rev 54: 113-161

Davis WJ, Mpitsos GJ, Siegler MVS, Pinneo JM, Davis KB (1974) Neuronal substrates of behavioral hierarchies and associative learning in Pleurobranchaea. Am Zool 14: 1037-1050

Deliagina TG, Zelenin PV, Orlovsky GN (2002) Encoding and decoding of reticulospinal commands. Brain Res Rev 40: 166-177

Edwards DH, Heitler WJ, Krasne FB (1999) Fifty years of a common neuron: the neurobiology of escape behavior in the crayfish. Trends Neurosci 22: 153-161

Grillner S (2006) Biological pattern generation: the cellular and computational logic of network in motion. Neuron 52: 751-766

Kagaya K, Takahata M (2006) Brain mechanisms controlling the spontaneous walking of crayfish. Zool Sci 23: 1202

Katz PS, Hooper SL (2007) Invertebrate central pattern generators. In "Invertebrate Neurobiology" Ed by G North, RJ Greenspan, Cold Spring Harbor Laboratory Press, Cold Spring Harbor, pp 251-279

Kien J (1990) Neuronal activity during spontaneous walking. 1. Starting and stopping. Comp Biochem Physiol A 95: 607-621

Kitagawa G, Akaike H (1978) A procedure for the modeling of nonstationary time series. Ann Inst Stat Math B 30: 351-363
Kristan W, Gillette R (2007) Behavioral choice. In "Invertebrate Neurobiology" Ed by G North, RJ Greenspan, Cold Spring Harbor Laboratory Press, Cold Spring Harbor, pp 533-553

Kristan WB Jr, Calabrese RL, Friesen WO (2005) Neuronal control of leech behavior. Prog Neurobiol 76: 279-327

Le Bon-Jego M, Masante-Roca I, Cattaert D (2006) Statedependent regulation of sensory-motor transmission: role of muscarinic receptors in sensory-motor integration in the crayfish walking system. Eur J Neurosci 23: 1283-1300

Marder E, Bucher D, Taylor AL (2005) Invertebrate central pattern generation moves along. Curr Biol 15: 685-699

Maye A, Hsieh C, Sugihara G, Brembs B (2007) Order in spontaneous behavior. PLoS One, May 16. DOI number 10.1371/ journal.pone. 0000443

McFarland D (1999) Animal Behavior. 3rd ed, Pearson Education Limited, Harlow

Moore PA, Scholz N, Atema J (1991) Chemical orientation of lobsters, Homarus americanus, in turbulent odor plumes. J Chem Ecol 17: 1293-1307

Mori S, Sakamoto T, Takakusaki K (1991) Interaction of posture and locomotion in cats: its automatic and volitional control aspects. In "Neurobiological Basis of Human Locomotion" Ed by $M$ Shimamura, S Grillner, VR Edgerton, Japan Scientific Societies Press, Tokyo, pp 21-32

Orlovsky GN, Deliagina TG, Grillner S (1999) Neural Control of Locomotion. From Mollusc to Man. Oxford University Press, London

Pearson KG, Ramirez J (1997) Sensory modulation of patterngenerating circuits. In "Neurons, Networks, and Motor Behavior” Ed by PSG Stein, S Grillner, Al Selverston, DG Stuart, MIT Press, Cambridge, MA, pp 225-235

Ramirez JM, Pearson KG (1988) Generation of motor patterns for walking and flight in motoneurones supplying bifunctional muscles in the locust. J Neurobiol 19: 257-282

Robertson RM (1986) Neuronal circuits controlling flight in the locust: central generation of the rhythm. Trends Neurosci 9: 278-280

Robertson RM, Pearson KG (1985) Neural circuits in the flight system of the locust. J Neurophysiol 53: 110-128

Schultz W (1999) The primate basal ganglia and the voluntary control of behaviour. In "The Volitional Brain" Ed by B Libet, A Freeman, K Sutherland, Imprint Academic, Exeter, pp 31-45

Sirota MG, Di Prisco GV, Dubuc R (2001) Stimulation of mesencephalic locomotor region elicits controlled swimming in semiintact lampreys. Eur J Neurosci 12: 4081-4092

Weissburg M (1997) Chemo- and mechanosensory orientation by crustaceans in laminar and turbulent flows: from odor trails to vortex streets. In "Orientation and Communication in Arthropods" Ed by M Lehrer, Birkhäuser Verlag, Basel, 215-246

Weissburg MJ, Zimmer-Faust RK (1993) Life and death in moving fluids: hydrodynamic effects on chemosensory-mediated predation. Ecology 74: 1438-1443

Weissburg MJ, Zimmer-Faust RK (1994) Odor plumes and how blue crabs use them in finding prey. J Exp Biol 197: 349-375

Wiersma CAG (1947) Giant nerve fiber system of the crayfish. A contribution to comparative physiology of synapse. J Neurophysiol 10: 23-38

Yamane S, Takahata M (2002) Experimental modification of stepping course in spontaneously initiated locomotor behavior in the crayfish Procambarus clarkii Girard. J Comp Physiol A 188: 13-23

Zimmer-Faust RK, Finelli CM, Pentcheff ND, Wethey DS (1995) Odor plumes and animal navigation in turbulent water flow: a field study. Biol Bull 188: 111-116

(Received March 28, 2008 / Accepted May 29, 2008) 